\title{
Wasserabpressende Fördereinrichtung zur energieeffizienten Entwässerung von Schüttgut
}

\author{
Water extorting conveying device for energy efficient dewatering of bulk materials
}

\author{
Thomas Mauersberger* \\ Thomas Linke ** \\ * fömat $\mathrm{GmbH}$ \\ Fördertechnik und Maschinenbau \\ ** Technische Universität Chemnitz \\ Institut für Fördertechnik und Kunststoffe \\ Professur Fördertechnik
}

$\mathbf{F}$ ür die Optimierung und somit Energieeinsparung beim Trocknen von Grüngut wurde eine mechanische Vorentwässerung entwickelt. Dieser Schritt wurde notwendig, da sich die politischen und ökonomischen Rahmenbedingungen, durch Subventionskürzungen und steigenden Energiepreisen, zu Ungunsten der Trocknungsbetriebe verändert haben und eine rein thermische Trocknung kaum noch rentabel ist. In einem gemeinsamen Kooperationsprojekt zwischen der TU Chemnitz und der fömat GmbH entstand eine Schneckenpresse speziell für Grüngut ohne dabei wichtige Nährstoffe beim Entwässern mit auszuspülen. - Seit 2010 ist diese Fördereinrichtung in den Trocknungsprozess vollständig integriert und es konnten während einer vollständigen Trocknungssaison in ca. 1.800 Betriebsstunden mehr als $10.500 \mathrm{~m}^{3}$ Wasser aus $47.200 \mathrm{t}$ Grüngut abgepresst werden. Dies hatte zur Folge, dass eine Energieeinsparung von über $25 \%$ erreicht wird. Die Qualität des Grünfutters wurde dabei nicht beeinträchtigt und die wichtigen Nährstoffe blieben im Grüngut nahezu vollständig erhalten.

[Schlüsselwörter: Schüttgut, Trocknung, Futtermittel, mechanische Vorentwässerung, Schneckenpresse]

$\mathbf{F}$ or optimizing and therefore energy saving while drying of green cuttings a mechanical predewatering machine was developed. This was necessary because of changes in the political and economic environment. Since subsidies have been reduced and the price of energy has been raised, pure thermal drying becomes unprofitable. A cooperation project between the Chemnitz University of Technology and the fömat GmbH resulted in a worm extruder especially for green cuttings with the advantage not rinsing out nutritive substances. - Since 2010 these conveying device has been fully integrated into the drying process.
During one drying season with approximately 1800 hours of operation the system was able to squeeze $10500 \mathrm{~m}^{3}$ water out of $47200 \mathrm{t}$ of green cuttings. In consequence $25 \%$ of energy was saved. The quality of green fodder was not impaired and all important nutrients were almost completely preserved.

[Keywords: bulk materials, drying, feeding stuff, mechanical pre-dewatering, worm extruder]

\section{PRoblemATIK DER THERMISCHEN TROCKNUNG VON FASERIGEM SCHÜTTGUT (GRÜNSCHNITT)}

Das Trocknen von Grüngut für Futterzwecke ist eine schonende und daher wichtige Form der Konservierung von Futtermittel. Durch die Wärmebehandlung beim Trocknen gelangt mehr Futtereiweiß des Grünguts in die Verdauungsorgane der Tiere und wird nicht vorher im Pansen abgebaut. Der hohe Gehalt an diesem nutzbaren Rohprotein, über 40\%, steigert die Milchleistung und verkürzt die Mastzeiten. Trockengrün stellt somit eine gute heimische Alternative zu importierten Soja und zu Kraftfutter dar [Goe10]. Es enthält zudem einen höheren Betakarotin-Gehalt und ist gegenüber Soja GVO-frei. Gerade der Aspekt der ökologischen und gesundheitsbewussten Ernährung und die Unabhängigkeit von Importpreisen der Futtermittel wie Soja lassen das Trockengrün für viele Landwirte attraktiver werden.

Vorteile von Trockengrün im Überblick [VST12]:

- Wirtschaftlich:

○ Geringste Konservierungsverluste

○ niedrige Kosten je Nährstoffeinheit

○ betriebseigenes, fütterungsfertiges Kraftfutter 
○

einfache Ernteverfahren, Lagerung und Fütterung

○ Rohproteinertrag pro Hektar 3x höher als Soja

- $\quad$ Tiergerecht

○ Hoher Anteil an nXP und APD

○ geschütztes, pansenstabiles Protein

○ hoher Gehalt an Polyensäuren (Linolsäure)

○ optimales Aminosäuremuster

○ Vitamine (E,K,B-Komplex)

○ hoher, stabiler Gehalt an Betakarotin

- Ökologisch

○ Einheimische Futtermittel

○ kurze Transportwege

○ Trockengras ersetzt importiertes Sojaprotein

- GVO-frei

○ aus Rauhfutter wird Kraftfutter

Bei allen Vorteilen des Trockengrüns stellt sich die Trocknung selbst als ein großer Nachteil dar. Aufgrund des hohen Wassergehalts des frischen Grünschnitts ist ein sehr großer Energiebedarf beim Trocknen notwendig. Die benötigte Energie wird größtenteils aus fossilen Brennstoffen gewonnen, wird zunehmend teurer und belastet die Umwelt. Für die Grünfuttertrocknung werden jährlich etwa 526.000 t Rohöläquivalent fossilen Brennstoffs benötigt. Dies führt zu einem jährlichen Kohlendioxidausstoß von 1,62 Mio. t [NoW10]. Um die Energiekosten zu decken, stellte die Europäische Union bisher Subventionen in Form einer Trockenfutterbeihilfe bereit. Damit die Herstellung von Trockenfutter auch ohne EU-Beihilfe wirtschaftlich erfolgen kann, müssen neue Lösungen zum Trocknen gesucht werden.

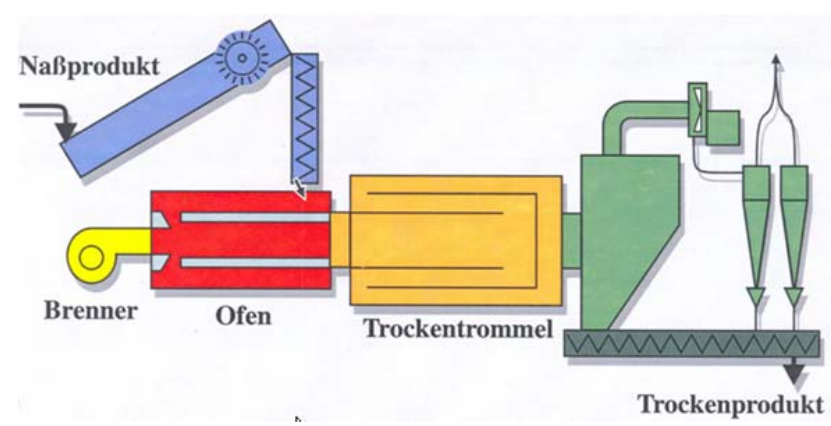

Abbildung 1. Vereinfachtes Schema zum Trocknungsprozess [LTD12]

Eine Umstellung des Energieträgers, weg vom Erdgas oder Flüssiggas hin zu einer $\mathrm{CO}_{2}$-neutralen Befeuerung mit Holzhackschnitzeln und anderer Biomasse, ist in einigen Trocknungsanlagen bereits erfolgreich realisiert. Andere Trocknungen versuchen über günstigere fossile Brennstoffe ihre Wirtschaftlichkeit beizubehalten. Dabei gibt es jedoch verstärkten Widerstand in der Bevölkerung (bspw. Trocknung Wolfratshausen Ost, Umstellung der Befeuerung auf Braunkohlestaub).

Um die Wirtschaftlichkeit sicher zu stellen, führt ein zweiter Ansatz über die Optimierung des Trocknungsprozesses. Eine einfache Möglichkeit besteht darin, das geschnittene Grüngut auf dem Feld vorwelken zu lassen. Dieser Vorgang reduziert den Wassergehalt enorm (siehe Tabelle 1), ist allerdings wetterabhängig. Das Gras kann nur an trockenen Tagen geschnitten werden und somit nicht immer bei idealem Wuchs. Auf Grund des Klimas ist dies für deutsche Trocknungen ein ungeeignetes Vorgehen. Zudem kommt es zu einer sehr ungleichmäßigen und damit unwirtschaftlichen Anlagenauslastung.

Tabelle 1. Wasser- und Feuchtegehalt ausgewählter Schüttgüter

\begin{tabular}{|l|c|c|}
\hline $\begin{array}{l}\text { Art/Zustand } \\
\text { des Grünguts }\end{array}$ & $\begin{array}{l}\text { Wassergehalt } \\
\text { U in [\%-WG] }\end{array}$ & $\begin{array}{c}\text { Feuchtegrad } \\
\text { X in [\%-FG] }\end{array}$ \\
\hline $\begin{array}{l}\text { Grüngut } \\
\text { (frisch ge- } \\
\text { schnitten) }\end{array}$ & $80-70$ & $400-233$ \\
\hline $\begin{array}{l}\text { Grüngut (vor- } \\
\text { gewelkt) }\end{array}$ & $65-40$ & $186-67$ \\
\hline Futtercobs & $12-11$ & $15-14$ \\
\hline
\end{tabular}

\section{ZIELE DES FORSCHUNGSVORHABENS}

In den Jahren 2009 bis 2011 wurde gemeinsam zwischen der TU Chemnitz und der fömat GmbH unter Mitwirkung der Trocknungsgenossenschaft Wolfratshausen Ost e.G. an einem Forschungsvorhaben gearbeitet. Ziel des Vorhabens war die Entwicklung einer mechanischen Vorentwässerung zur schonenden Reduzierung des Wassergehalts im frisch geschnittenen Grüngut.

Dabei sollten folgende Kriterien erfüllt werden:

- Entwässerung ohne Entzug wichtiger Nährstoffe

- hohe Anlagenverfügbarkeit

- Integrationsmöglichkeit in bestehende Trocknungsanlagen

- $\quad k o n t i n u i e r l i c h e ~ A r b e i t s w e i s e$

- $\quad$ einfache Zugänglichkeit für Reinigung, Wartung und Instandhaltung

- $\quad$ einfaches, robustes Maschinenkonzept

- Witterungsunabhängigkeit

- $\quad$ Förderleistung 25 t/h frisches Grüngut mit ca. 80 bis $85 \%$ Wassergehalt 


\section{UMSETZUNG}

Für die Realisierung eines Prototypen wurden verschiedene Wirkpaarungen zur mechanischen Entwässerung analysiert und bewertet. Als Vorzugsvariante wurde eine Schneckenpresse gewählt, welche in ähnlicher Form schon Anwendung in der Entwässerung von Recyclingabfall findet oder auch zur Entwässerung von Grünschnitt genutzt wird, jedoch mit dem Ziel der maximalen Entwässerung und anschließender thermischer Verwertung.

Im folgenden Verlauf wurde ein Demonstrator entwickelt mit dessen Hilfe die Funktionstüchtigkeit geprüft sowie Aussagen zur Leistungsaufnahme und Fördermenge getroffen werden konnten.

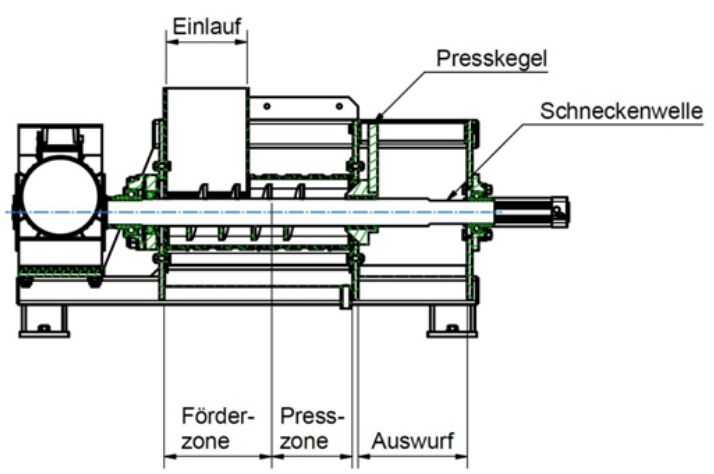

Abbildung 2. Demonstrator

Um eine möglichst einfache Konstruktion zu erhalten, wurde die Schneckenpresse mit einem zylindrischen Schneckentrog in Schalenbauweise und einer einfachgewendelten Schneckenwelle mit gleichbleibender Steigung sowie gleichbleibendem Kernrohr ausgeführt. Der nötige Druck zur Entwässerung wird über einen auf der Welle axial verschiebbar gelagerten Presskegel erzeugt. Damit kann über den Austrittsspalt zwischen Kegel und Schneckentrog der Pressdruck geregelt werden.

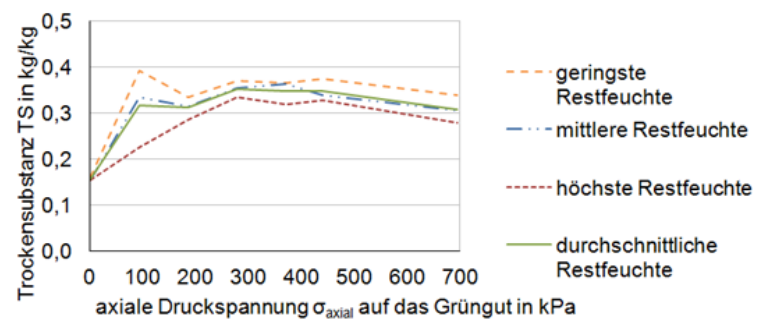

Abbildung 3. Abhängigkeit der Restfeuchte von der axialen Druckspannung

Nach den ersten Entwässerungsversuchen konnten Aussagen bezüglich der benötigten Antriebsleistungen und Druckspannungen gemacht werden (siehe Abbildung 3). Bei einer Analyse des vorentwässerten Grünguts hat sich gezeigt, dass die Inhaltsstoffe nahezu vollständig er- halten blieben und der Wassergehalt von durchschnittlich 80 bis $85 \%$ auf unter $70 \%$ verringert werden konnte. (In absoluten Zahlen: aus $10 \mathrm{~kg}$ Grüngut mit 80\% Feuchtegehalt werden ca. 3,3 l Wasser mechanisch entzogen)

Die Ergebnisse am Demonstrator flossen in die Entwicklung einer Pilotanlage für die Trocknungsgenossenschaft Wolfratshausen Ost e.G. ein (siehe Abbildung 4).

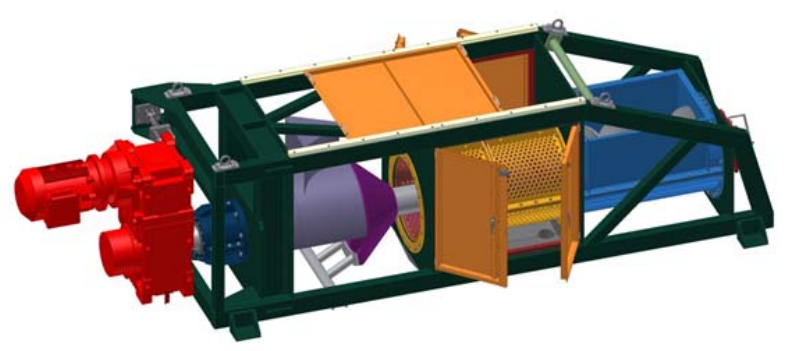

Abbildung 4. CAD-Modell: Pressschnecke zur Vorentwässerung

Wie beim Demonstrator wurde die Konstruktion des Troges der Pilotanlage (siehe Abbildung 5) in einer verschraubten Schalenbauweise ausgeführt. Es hat sich während der Versuche immer wieder gezeigt, dass sich das Grüngut extrem verdichtet und es zu Verfestigungen kommen kann oder das Gut bei zu hohen Drücken mitdreht und nicht mehr gefördert wird. Durch die Schalenbauweise ist es im äußersten Notfall möglich, den Trog zu demontieren um Verfestigungen zu lösen. Um die Förderung zu verbessern wurden zusätzlich Bleche, welche in die Förder- und Presszone hineinragen, zwischen den Trögen montiert, welche das Mitdrehen verhindern. Im Pressbereich sind die Schalen als Doppelsiebe ausgebildet um einen idealen Austritt des Presssaftes zu gewährleisten.

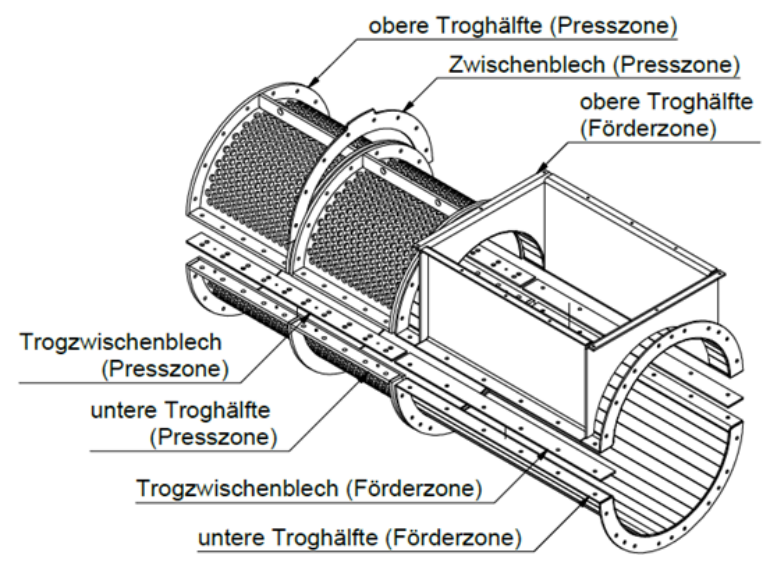

\section{Abbildung 5. Trogaufbau}

Die Schneckenwelle wurde im Gegensatz zum Demonstrator 2-gängig ausgeführt, um eine gleichmäßigere Kraftverteilung zu erreichen. Zusätzlich wurden die Schneckenflügel mit einer verschleißfesten Schicht im Pressbereich versehen. 
Bei dem Presssaft handelt es sich um ein sehr aggressives Medium. Aus diesem Grund sind produktberührende Teile in Edelstahl und alle Lager mit Mehrfachdichtungen ausgeführt worden.

\section{ERGEBNISSE}

Nach einer Trocknungssaison mit der Pilotanlage konnten enorme Einsparungen ermittelt werden. Während der Saison 2010 konnten $10.500 \mathrm{~m}^{3}$ Wasser aus $47.200 \mathrm{t}$ Grüngut (siehe Abbildung 6) mit einer durchschnittlichen Eingangsfeuchte von 78,6 \% mechanisch entzogen werden. Dabei wurde eine durchschnittliche Ausgangsfeuchte von ca. 72,4 \% erreicht. Die Einsparungen an Energiekosten betragen ca. $20 € / \mathrm{m}^{3}$, somit ca. $210 \mathrm{~T} €$ für die Saison 2010. Der Ausstoß an $\mathrm{CO}_{2}$ wurde um ca. $26 \%$ verringert.

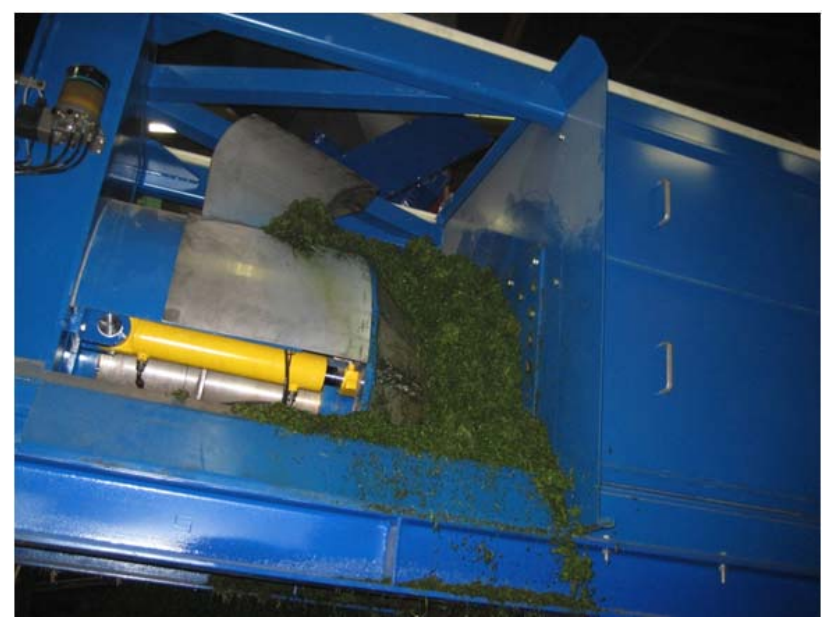

Abbildung 6. Auswurf vorentwässertes Grüngut

Neben den Hauptvorteilen der mechanischen Vorentwässerung haben sich noch einige weitere Vorteile gezeigt:

- Verkürzung der Anlagenlaufzeit bzw. höhere Förder-/ Trocknungsleistung möglich

- nahezu witterungsunabhängige Ernte

- geringerer Verschleiß an nachfolgenden Förderern

- $\quad$ besserer Materialfluss nach der Vorentwässerung

- Teilweise Auswaschung von Sand und Schmutz beim Entzug des Haftwassers

- $\quad$ Senkung der Trocknungsofen-Eingangstemperatur von $750-900^{\circ} \mathrm{C}$ auf $450-600^{\circ} \mathrm{C}$

- Presssaft kann auf landwirtschaftliche Nutzfläche wieder ausgebracht werden

- Durch die mechanische Vorentwässerung wurde eine Energieeinsparung von 25\% bei nur minimalen und vernachlässigbaren Qualitätsverlusten der Trockengrünqualität erreicht. In 2011 wurde eine weitere noch leistungsfähigere Anlage in einem größeren Trocknungsbetrieb in MecklenburgVorpommern in Betrieb genommen (siehe Abbildung 7). In den nächsten Jahren ist die Realisierung weiterer Anlagen geplant.

\section{ZUSAMMENFASSUNG}

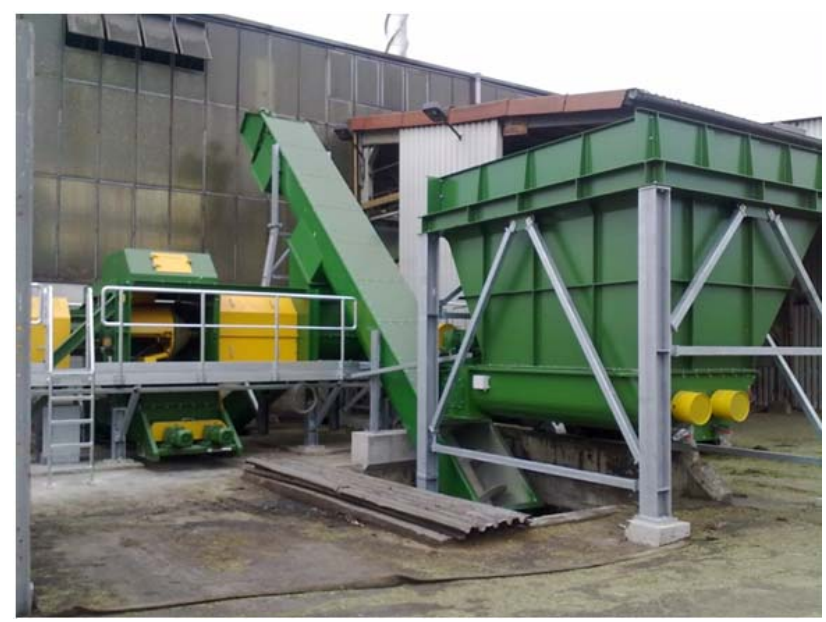

Abbildung 7. Leistungsfähigere und überarbeitete Anlage in Mecklenburg-Vorpommern

Das Kooperationsprojekt KF2305201KO9 der TU Chemnitz und fömat GmbH wurde über die AiF im Rahmen des Programms "Zentrales Innovationsprogramm Mittelstand" (ZIM) vom Bundesministerium für Wirtschaft und Technologie aufgrund eines Beschlusses des Deutschen Bundestages gefördert.

\section{LITERATURVERZEICHNIS}

[Goe10] Götz, Michael: Gras mit Hackgut trocknen. Internetpräsentation, Bundesministerium für Land- und Forstwirtschaft, Umwelt und Wasserwirtschaft (BMLFUW) Österreich, 2010, LANDnet, http://www.bmlfuw.gv.at/article/ articleview/77064/1/4984, 01/2010

[VST12] Informationsbroschüre „Trockengras ist top...". Verband Schweizerischer Trocknungs-Betriebe. 2012

[NoW10] N.N.: Aktuelle News. Internetpräsentation, Qualitäts- und Produktionssicherung Nord-West GmbH, http://www.iq-agrar. org/eukommission_rechtfertigt_geplante_ entkopplung_der.html, 01/2010.

[LTD12] Vereinfachtes Schema Trocknungsprozess, Bundesfachverband Landwirtschaftlicher Trocknungswerke Deutschland e.V., 2012, http://www.bltdtrockengruen.de, 07/2012 